\title{
Study on crystallization kinetics of binary alloys through model colloidal mixtures
}

\author{
Yanping Hu, Ding Lan, Guoliang Dai, Heng Jiang, Li Duan, BingChen Wei* \\ Key Laboratory of Microgravity (National Microgravity Laboratory), Institute of Mechanics, Chinese Academy of Sciences, Beijing 100190, PR China
}

\section{A R T I C L E I N F O}

\section{Article history:}

Received 2 September 2009

Received in revised form 8 February 2010

Accepted 19 February 2010

Available online 3 March 2010

\section{Keywords:}

Glass-forming ability

Metallic glass

Colloid

Crystallization

\begin{abstract}
A B S T R A C T
Since the discovery of amorphous alloys, extensive attentions have been paid to understand the mechanism of glass-forming. The structural studies on its underlying mechanism have met challenge on direct structural characterization. It has been shown that the phase behavior of colloids dispersed in a solvent is thermodynamically equivalent to that of atoms and small molecules, however, colloids can be studied with optical microscopy due to their relatively large size. In this work, we use a binary colloidal model system with the particle size ratio comparable to atomic ratio of reported binary bulk metallic glasses to study the topological effect on crystallization and glass-forming ability of binary metallic alloys (Cu-Hf and $\mathrm{Cu}-\mathrm{Zr}$ systems). The crystallization kinetics and structure of the colloid system were studied by realtime optical examination and light scattering technique. The results exhibit that there are two confined regions in the mixing ratio (composition) range in the colloid system with an enhanced glass-forming ability and retarded crystallization kinetics. The agreement between results of the model system and the experimental data on the binary bulk metallic glass formation suggests that purely topological factor plays an important role in determining the glass-forming ability.
\end{abstract}

(C) 2010 Elsevier B.V. All rights reserved.

\section{Introduction}

When a liquid is cooled sufficiently, it may undergo dynamical arrest and become a glass, if the liquid is stable enough. Atomic structures in liquids are believed to have an intrinsic influence on the glass-forming ability (GFA) of alloy [1]. However, it is still hard to identify the structural mechanism responsible for the competition between crystallization and vitrification, as the characterization of the atomic-level structure in liquid and glass state is very challenging for experimental techniques, such as X-ray scattering which usually give a statistical result. Despite this, structural studies have ascribed the high GFA to a complicated dense random packed structure, which lead to high viscosity of the supercooled liquid state and slow crystallization [1-4]. The select of multicomponents on bulk metallic glass (BMG) formation is concluded as 'a principal confusion' [5]. This compositional complexity makes it extremely difficult for different atoms to rearrange their spatial positions so that they are frozen directly into a disordered glassy structure upon cooling. Recently, binary BMG forming alloys have also been developed in a few alloy families [6-14], and their GFA is found to be very sensitive to the minor change of chemical composition $[15,16]$. These further confuse the criterions of GFA in metallic alloys. The degree of atomic arrangement is governed by two fac-

\footnotetext{
* Corresponding author. Tel.: +86 10 82544118; fax: +86 1082544096 .

E-mail address: weibc@imech.ac.cn (B.Wei).
}

tors: atomic packing and atomic bonding. Atomic packing involves some geometrical parameters, such as atomic size difference and size of clusters, while atomic bonding is more related to the electronic structures. It is still unclear that the effect of compositional complexity on structure is purely topological $[1,17]$ or is strongly influenced by the intricate bonding environments $[4,18]$.

Suspensions of spherical colloidal particles in a liquid show a fascinating variety of phase behavior which are recognized as mesoscopic 'model atoms' because they have a well-defined thermodynamic temperature, and also form glasses [19-21]. Furthermore, it was shown by computer simulations and experiments that such a system shows a purely entropy-driven phase transition from a disordered fluid phase to a crystal phase under the appropriate conditions [22]. A significant breakthrough thus came as colloids can be studied with optical microscopy at the single particle level. It is clear that the use of binary colloid systems can significantly inhibit crystallization, and may be a fruitful avenue for exploration of the competition between crystallization and vitrification $[23,24]$. In this work, we use a binary colloidal system with the size ratio comparable to that of the reported binary BMG forming alloys, to provide a visible experimental approach for studying the compositional effect (geometrical aspect) of crystallization kinetics and GFA.

\section{Experimental}

The particles used in these experiments were polystyrene spheres, with average radius of $2.0 \mu \mathrm{m}$ and $2.9 \mu \mathrm{m}$, respectively, which was examined by scanning electron 


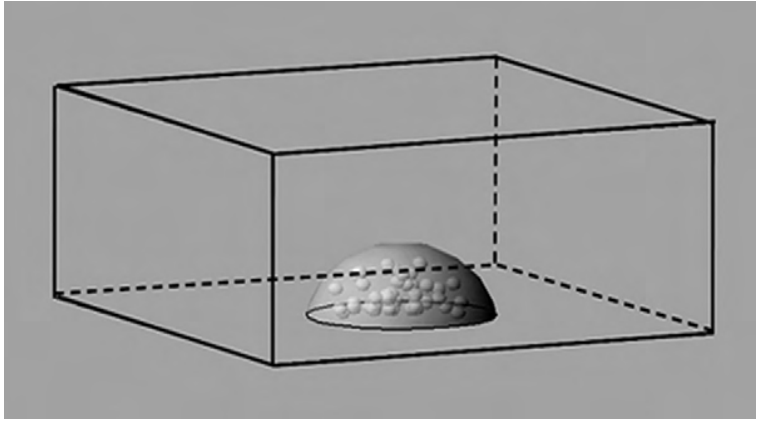

Fig. 1. Schematic figure of the sample for optical real-time observation.

microscopy (SEM). The size polydispersity of both was less than 0.05 . The particles were dispersed in Milli-Q water without ion exchange resin. Because of the large size of PS particles, they behaved a nearly hard spheres feature [25]. These two kinds of PS spheres were fully mixed with different particle numbers, with mixing ratio, which is defined as $f_{\mathrm{m}}=N_{\text {small }} / N_{\text {larger }}$, from 0.6 to 2.2 , increasing by a step of 0.2 . The volume fraction for all the samples is identical, $10 \%$, an obvious liquid state of colloidal system.

The suspensions of mixture were made into small droplets with radius range from 0.1 to $1 \mathrm{~mm}$, and injected into a cell with size of $18 \mathrm{~mm} \times 18 \mathrm{~mm} \times 1 \mathrm{~mm}$, then covered every single droplet with a thin membrane of silicone oil. The cell was sealed for optical observation. Fig. 1 shows the schematics of the optical cell with a single drop of the mixture inside.

The existence of the thin membrane of silicone oil enabled the water phase in the mixture droplets to vaporize into the optical cell in a very slow speed, so that particles inside the droplets had enough time to self-assemble into a locally favorable structure.

After the final structure had been obtained judging from the optical microscope, the optical cell was set in a light channel to obtain the characteristic information of structure by light scattering technique. The light source used here is a He/Ne laser with the wavelength of laser beam $532 \mathrm{~nm}$.

\section{Results and discussion}

In order to check the validity of the present method, we firstly consider the phase behavior of the monodisperse colloid system in the cell by optical microscopy. The initial states (liquid state) of the monodisperse colloid mixture with particle size of 2.0 and $2.9 \mu \mathrm{m}$ is shown in Fig. 2a and c, respectively. It has been shown that a smooth hard wall causes pronounced layering and pre-freezing of the fluid phase at the wall $[26,27]$. A stable crystal can be grown, where the particles are stick-up by the strong capillary forces. If the volume fraction of particles is appropriately controlled, one layer crystal, i.e. two-dimensional (2D) crystals can be formed as shown in Fig. $2 b$ and d. It can be seen that the small particles froze into a few crystals, while the large particles formed a single crystal. The time duration from the initial liquid state to the final frozen state is $138 \mathrm{~min}$ and $220 \mathrm{~min}$ for the small and large particles, respectively. Although the $2 \mathrm{D}$ results cannot provide a truly quantitative measurement, they do yield information about the general structure, and can easily demonstrate a long-range ordered (crystalline) structure. In addition, the pre-freezing on the container wall could resemble the condition of heterogeneous nucleation of crystals, which is a key factor dominating the glass formation.

The atomic size ratio $\left(q=r_{\text {small }} / r_{\text {large }}\right)$ of several reported binary metal-metal type BMG systems is listed in Table 1. It can be found

Table 1

Atomic size ratio $\left(r_{\text {small }} / r_{\text {large }}\right)$ of several reported binary metallic glasses systems.

\begin{tabular}{ll}
\hline Alloy system & $r_{\text {small }} / r_{\text {large }}$ \\
\hline $\mathrm{Cu}-\mathrm{Zr} \mathrm{[10-12]}$ & 0.7039 \\
$\mathrm{Cu}-\mathrm{Hf} \mathrm{[7-9]}$ & 0.6971 \\
$\mathrm{Ni}-\mathrm{Nb}[13]$ & 0.7525 \\
$\mathrm{Ca}-\mathrm{Al} \mathrm{[14]}$ & 0.6082 \\
\hline
\end{tabular}

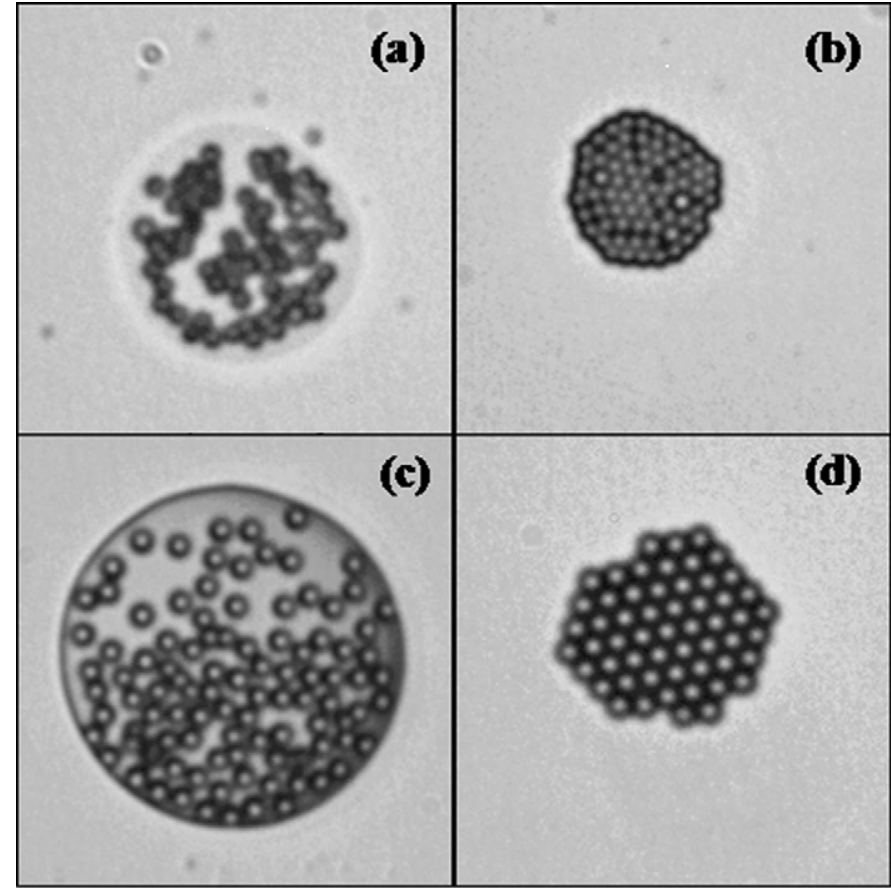

Fig. 2. Optical micrograph of monodisperse colloidal systems: (a) the initial liquid state of colloidal suspensions with particle size $2.0 \mu \mathrm{m}$; (b) the frozen state of (a) (c) the initial liquid state of colloidal suspensions with particle size $2.9 \mu \mathrm{m}$; (d) the frozen state of $(\mathrm{c})$.

that $q$ ranges from 0.55 to 0.80 . Here, we use a binary colloid system to simulate the situation of binary BMGs with large particle $(d=2.9 \mu \mathrm{m})$ and smaller particles $(d=2.0 \mu \mathrm{m})$. The size ratio of small to large particles $q$ is thus 0.69 , which is comparable to that in $\mathrm{Cu}-\mathrm{Zr}$ (0.7039), and especially in $\mathrm{Cu}-\mathrm{Hf}(0.6971)$ system.

Like the case in monodisperse colloid system, particles in the binary system also tend to self-assembly, resulting in the volume exclusion. However, the binary system has complicated frozen solid phases, which are sensitive to the mixing ratio (composition). The optical freezing structures as functions of composition are shown in Fig. 3. In contrast to the case in the monodisperse system, the binary colloidal systems do not freeze into a distinct crystalline phase, but form an irregular structure. Partially ordered or distorted ordered structures can be seen at some mixing rations $\left(f_{\mathrm{m}}\right)$, e.g. at $f_{\mathrm{m}}=0.6$, $0.8,1.4,1.6$. In addition, phase separation can clearly be found at $f_{\mathrm{m}}=0.8$ and 1.4 , wherein large particles tend to segregation at the outer boundary of the mixture. A highly disordered structure is formed at $f_{\mathrm{m}}=1.2$ and 1.6 .

Light scattering technique was used to further study the structure of the frozen monodispere and binary mixtures. The setup of the light scattering measurement is shown in Fig. 4a. For the monodisperse system, clear Bragg spots can be captured, as seen in Fig. 4b indicating the order structures. Typical light scattering results for binary systems are shown in Fig. 4c and d. No distinct Bragg spots are found any more, demonstrating the lack of longrange order. These results agree with the direct optical observation 2D structures in Fig. 3. Furthermore, there is still difference in the scattering pattern among the binary systems at various $f_{\mathrm{m}}$. The mixtures with $f_{\mathrm{m}}=0.6,0.8,1.0,1.4,1.6,2.0$ exhibit a streak-like pattern, implying the existence of ordered clusters within the solid phases, as shown in Fig. 4c. Whereas, mixtures with $f_{\mathrm{m}}=1.2$ and 1.8 exhibit a featureless scattering pattern, implying a disordered structure, like the case in the glassy state of condensed matter. The detailed structure factor of the frozen mixtures and its relationship with the glass formation were not performed in the present work. Instead, we use 




Fig. 3. The frozen structures of suspensions of different mixing ratios $f_{\mathrm{m}}=0.6$ (a), 0.8 (b), 1.0 (c), 1.2 (d), 1.4 (e), 1.6 (f), 1.8 (g), and 2.0 (h).

the time scale from the initial liquid suspensions to the freezing state (when particles do not change their position) to demonstrate the stability of liquid state at various mixing ratios.

The time scale of liquid stability as function of the mixing ratio is plotted in Fig. 5. It should be noted that as mixing ratio increases from 0.4 to 2.2, time for reaching freezing state exhibits a two-peak curve. The two sharp time peaks correspond to the mixing ratio of 1.2 and 1.8 , respectively. The time scale at $f_{\mathrm{m}}=1.2$ and 1.8 is $690 \mathrm{~min}$ and 730 min respectively, which is about 2.7 times longer than the average time ( $\sim 260 \mathrm{~min}$.) of other mixing ratios. This means that the binary suspensions are more stable at these two compositions, wherein a sluggish arrangement of particles and slow kinetic of volume exclusion exist owing to the specific mixing ratio of the two kind of particles. This is in a good agreement with the optical structure (Fig. 3) and light scattering results (Fig. 4), and further prove that the packing of particles is difficult at $f_{\mathrm{m}}=1.2$ and 1.8 . The results suggest that for the present binary colloidal system there exist two confined regions in the mixing ratio/composition range accentuated by an enhanced glass-forming ability or retarded crystallization kinetics.
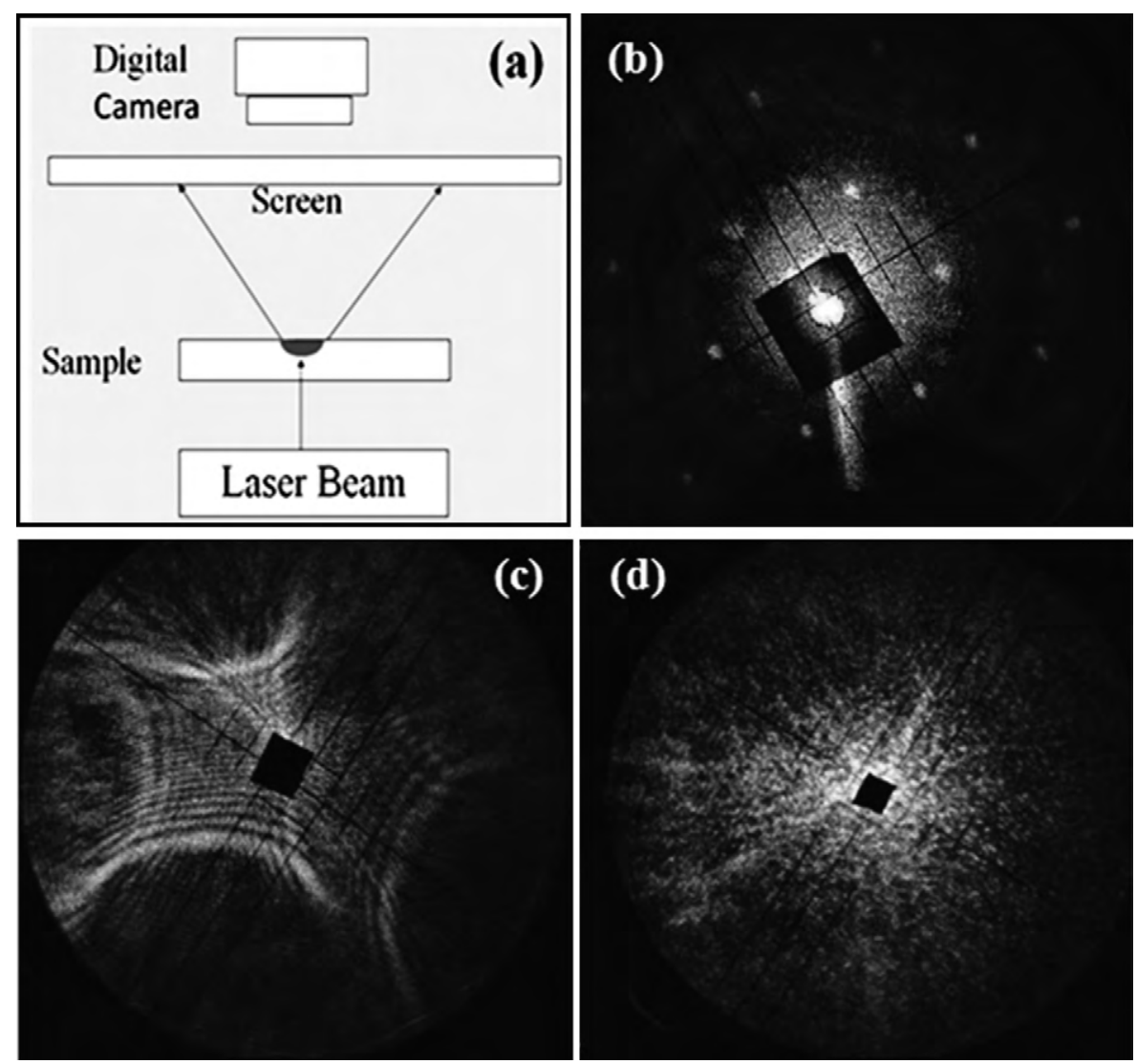

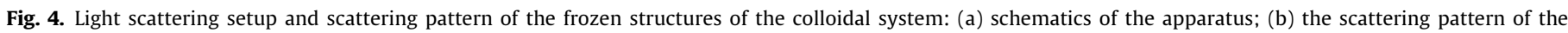

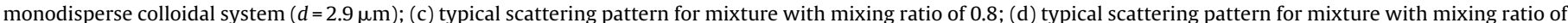
1.8 . 


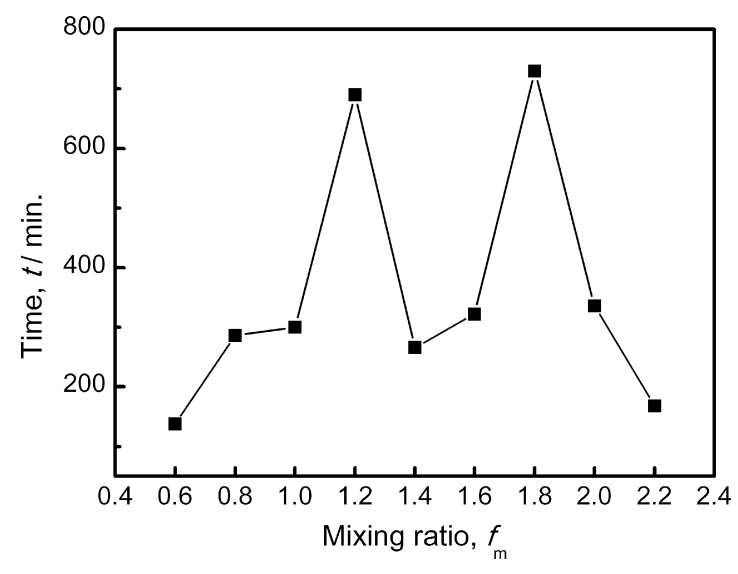

Fig. 5. The time scale of liquid stability as function of the mixing ratio.

The size ratio $(q)$ of small to large particles in the present binary colloid system is 0.69 , which is quite similar to the atomic size ratio of $\mathrm{Cu}-\mathrm{Hf}$ binary alloy (0.6971). It is interesting to find that there are two best glass-forming compositions reported up to now, $\mathrm{Cu}_{55} \mathrm{Hf}_{45}$ [9] and $\mathrm{Cu}_{65} \mathrm{Hf}_{35}[7,8]$ with the corresponding mixing ratios of 1.22 and 1.86 , respectively. The agreement between the colloid mixtures results and the experiment data of binary bulk metallic glasses supports the present experimental design, and gives a direct evidence for the validity of topological factor in determining the glass-forming ability in binary metal liquid. At certain mixed ratios (here, $f_{\mathrm{m}}=1.2$ and 1.8 , respectively), the alloy exhibits a sluggish crystallization kinetic as shown is Fig. 5, possibly due to the more effective atomic packing. The correlation between the atomic packing geometry and mixed ration will be investigated later.

The CuZr binary alloy has an atomic ratio of 0.7039 , which is also close to the particle size ratio of the present binary colloid system. The best BMG forming compositions $\mathrm{Cu}_{64.5} \mathrm{Zr}_{35.5}$ [11] and $\mathrm{Cu}_{64} \mathrm{Zr}_{36}[10]$ correspond to a mixing ratio of 1.82 and 1.78 , respectively. This also agrees well with the colloid mixture exprimental results. The $\mathrm{CuZr}$ binary BMGs composition around $\mathrm{Cu}_{50} \mathrm{Zr}_{50}$ [12] (corresponding to the mixing ratio of 1.0 here) does not exhibit easy glass formation ablility in the colloid system. This may be due to the slight deviation of the particle size ratio from the atomic size ratio.

\section{Conclusions}

A binary colloid system with the two different particle sizes (2.0 and $2.9 \mu \mathrm{m}$, respectively) was prepared with the particle size ratio of 0.69 , which is comparable with the atomic size ratio of $\mathrm{Cu}-\mathrm{Hf}$ and $\mathrm{Cu}-\mathrm{Zr}$ binary alloys. A sluggish crystallization kinetics and good glass-forming ability are found at the particle mixing ratios of 1.2 and 1.8. The time duration from the initial liquid state to the final squeezed state shows a two-peak curve on the function of mixing ratio. Comparison of the present results with experimental data for binary bulk metallic glasses supports the validity of purely topological effect on the glass-forming ability of binary metallic melts.

\section{Acknowledgements}

This work is financially supported by the National Nature Science Foundation of China (grant nos. 50731008 and 50771102) and National Basic Research Program of China (973 Program, no. 2007CB613905).

\section{References}

[1] D.B. Miracle, T. Egami, K.M. Flores, K.F. Kelton, MRS Bull. 32 (2007) 629.

[2] D.E. Polk, Scripta Metall. 4 (1970) 117.

[3] R. Busch, J. Schroers, W.H. Wang, MRS Bull. 32 (2007) 620

[4] Y.Q. Cheng, E. Ma, H.W. Sheng, Phys. Rev. Lett. 102 (2009) 245501.

[5] A.L. Greer, Nature 366 (1993) 303.

[6] A. Inoue, Acta Mater. 48 (2000) 279.

[7] L. Xia, D. Ding, S.T. Shan, Y.D. Dong, J. Phys. : Condens. Matter 18 (2006) 3543.

[8] G. Duan, D.H. Xu, W. Johnson, Metall. Mater. Trans. A 36 (2005) 456

[9] A. Inoue, W. Zhang, Mater. Trans. 45 (2004) 584.

[10] D.H. Xu, B. Lohwongwatana, G. Duan, W.L. Johnson, C. Garland, Acta Mater. 52 (2004) 2621.

[11] D. Wang, Y. Li, B.B. Sun, M.L. Sui, K. Lu, E. Ma, Appl. Phys. Lett. 84 (2004) 4029.

[12] M.B. Tang, D.Q. Zhao, M.X. Pan, W.H. Wang, Chin. Phys. Lett. 21 (2004) 901.

[13] L. Xia, W.H. Li, S.S. Fang, B.C. Wei, Y.D. Dong, J. Appl. Phys. 99 (2006) 026103.

[14] F.Q. Guo, S.J. Poon, G.J. Shiflet, Appl. Phys. Lett. 84 (2004) 37

[15] Y. Li, Q. Guo, J.A. Kalb, C.V. Thompson, Science 322 (2008) 1816

[16] G. Duan, M.L. Lind, M.D. Demetriou, W.L. Johnson, et al., Appl. Phys. Lett. 89 (2006) 151901.

[17] T. Egami, J. Non-Cryst. Solid 317 (2003) 30.

[18] Y.M. Wang, C.H. Shek, J.B. Qiang, et al., Scripta Mater. 48 (2003) 1525.

[19] P.N. Pusey, W. Megen, Nature (Lond.) 320 (1986) 340.

[20] W. Van Megen, S.M. Underwood, Nature 49 (1994) 4206.

[21] J.C. Conrad, P.P. Dhillon, E.R. Weeks, D.R. Reichman, D.A. Weitz, Phys. Rev. Lett 97 (2006) 265701.

[22] P.D. Kaplan, J.L. Rouke, A.G. Yodh, et al., Phys. Rev. Lett. 72 (1994) 582.

[23] H. Shintani, H. Tanaka, Nat. Phys. 2 (2006) 200.

[24] T. Kawasaki, T. Araki, H. Tanaka, Phys. Rev. Lett. 99 (2007) 215701.

[25] S. Sanyal, N. Easwar, S. Ramaswamy, A.K. Sood, Europhys. Lett. 18 (1992) 107.

[26] D.J. Courtemanche, F. van Swol, Phys. Rev. Lett. 69 (1992) 2078.

[27] M. Dijkstra, Phys. Rev. Lett. 93 (2004) 108303. 